\title{
LA RELIGIONE ROMANA IN PROSPETTIVA
}

\author{
Nota del m.s. JOHN SCHEID $(*)$
}

(Adunanza del 3 novembre 2016)

SUNTO. - È necessario mettere in discussione la confusione attualmente prevalente nello studio delle religioni antiche tra la religione dell'individuo, nel senso romantico, e il ritualismo politeistico praticato nel mondo romano fino all'avvento del cristianesimo. Rifiutando questo riferimento anacronistico alla nozione di individuo e includendo nella sua ricerca la storia della nozione di persona, che l'antropologia storica ha dimostrato essere un concetto emerso dal IV-V secolo e in un contesto cristiano, lo storico è molto più aperto allo studio e alla comprensione delle religioni altrui.

$$
* * *
$$

ABSTRACT. - It is necessary to question the confusion currently prevalent in the study of ancient religions between the religion of the individual, in the romantic meaning of the concept, and polytheistic and ritualistic religions as practiced in the Roman world until the emergence of Christianity. By refusing this anachronistic reference to the notion of the individual, and by including in his research the history of the notion of person, which, as historical anthropology has shown, is a later concept that emerged during the forth-fifth century and in a Christian context, the historian is much more open to the study and understanding of religions different from Christianity.

Nel corso dei miei studi avevo imparato che le religioni di Roma antica non conoscevano né rivelazione né libro rivelato, cioè né dogmi né autorità dogmatica superiore. Quando Claude Nicolet mi chiese,

(*) Professeur émérite du Collège de France. Membro straniero dell'Istituto Lombardo Accademia di Scienze e Lettere, Milano, Italy.

E-mail: john.scheid@college-de-france.fr 
nell'inverno 1977, di scrivere un capitolo sulla religione per un volume dedicato alle strutture della Repubblica romana, mi misi a riflettere su questo tema che, in quegli anni, era soltanto marginalmente presente nelle mie ricerche, ma sul quale avevo ricevuto all'Ecole pratique des hautes études un'eccellente formazione. Strada facendo, scoprii che a Roma la religione veniva praticata collettivamente; cioè nel quadro di gruppi sociali più o meno estesi, di cui le autorità temporali definivano la teologia e gli obblighi rituali. L'individuo praticava la sua religione secondo la sua posizione: in quanto cittadino partecipava passivamente o attivamente ai culti pubblici; in quanto magistrato celebrava i riti della religione pubblica; nel quadro della famiglia la sua partecipazione ai culti avveniva diversamente a seconda che fosse pater familias o membro della famiglia. Nelle associazioni il presidente annuale celebrava il culto e i membri vi partecipavano. Questo approccio era diffuso da qualche anno anche nello studio della religione greca in Francia, Inghilterra, negli Stati Uniti, e presso certi colleghi italiani. Questo permetteva di sfuggire all'influenza delle rappresentazioni cristiane della vita religiosa che si impongono nolens volens a ciascuno di noi. Questo modello di lettura, difeso da anni da Georges Dumézil e Jean-Pierre Vernant, p. es., si riferiva all'antropologia sociale. Proseguendo su questa strada scoprii, tramite lo studio dei rendiconti degli Arvali, un secondo elemento centrale nella vita religiosa antica: il rito. I Fratelli Arvali, un collegio sacerdotale senatoriale pubblico "riformato" da Ottaviano verso gli anni 31-28 a.C., celebravano ogni anno un sacrificio a Dea Dia, che garantiva la buona luce solare al momento della maturazione dei cereali, e praticavano altri riti pubblici legati alla salute dell'imperatore. Gli Arvali hanno lasciato frammenti epigrafici di rendiconti annuali delle loro azioni e decisioni, che costituiscono un'incomparabile fonte di conoscenza di prima mano sui riti romani. In religioni senza rivelazione, senza libro rivelato, la pietà era radicata negli obblighi rituali, che permettono oggi di ricostruire una parte dei diversi enunciati impliciti della pratica religiosa. Questo approccio inseriva le religioni greca e romana nel contesto delle innumerevoli religioni ritualistiche di cui ancora oggi i rituali costituiscono l'obbligo religioso essenziale, da quelle dei popoli dell'Africa o dell'Amazzonia fino a quelle dell'India vedica o indù, oppure, più vicino a noi, al giudaismo come lo definì Maimonide.

Ora, ormai da quindici anni, un tale modello interpretativo delle religioni antiche è stato messo in discussione, in Germania e in Gran 
Bretagna. In sé stessa questa critica sarebbe stata salutare, perché dopo cinquant'anni di ricerche su questa scia, era possibile migliorare, precisare o correggere diversi aspetti della teoria. Si poteva - e si doveva per esempio criticare il fatto che i riti fossero stati trascurati dagli studiosi, o che la maggior parte degli studi si fosse limitata ad Atene e Roma, o ai soli testi letterari e all'epoca classica ateniese e al periodo repubblicano romano. Invece, si trattava di un rifiuto totale della storia delle religioni antiche come era stata fatta a partire degli anni Trenta, e per certi particolari già dall'inizio del novecento, in stretto contatto con le fonti. Di fronte a questo rifiuto, si sarebbe potuto alzare le spalle e tornare al lavoro. Ma l'attacco mi pareva troppo globale e soprattutto destinato a sedurre i giovani. Decisi perciò di rispondere, in un libro, Les dieux, l'État et l'individu. Réflexions sur la religion civique à Rome, pubblicato nel 2013. In questa occasione tentai di rispondere alle idee dei decostruzionisti, mirando anche ai controsensi testuali e storici che esse comportavano per quanto riguarda le istituzioni. Tutta questa decostruzione mi pareva portare l'impronta retorica di dottrine legate alla Riforma e diffuse nell'ambito della storia antica nella Germania settentrionale dell'ottocento.

Uno degli argomenti di questa teoria mi sembrò immediatamente incongruente. Le critiche dell'approccio francese alle religioni dell'Antichità si fondavano infatti su un'opinione diffusa fra i filosofi dell'inizio dell'ottocento, che si ritrova nei manuali e libri di storia romana fino agli anni sessanta del novecento. Secondo questa opinione, la religione romana era in decadenza o addirittura morta fin dal III secolo a.C., quando essa si riduceva ad un ritualismo assurdo e privo di senso. L'argomento è ben noto. Ma i contradittori del modello interpretativo più diffuso delle religioni greca e romana utilizzano questo luogo comune della filosofia e della storia antica dell'ottocento, da Hegel a Mommsen e tanti altri, senza prendere in esame la critica esplicita del ritualismo da parte di questi teorici. Da parte di chi ha tentato di decostruire la storia delle religioni antiche quale si è scritta da Wissowa, Dumézil e Vernant in poi, dunque, il rifiuto generale del modello collettivo e ritualista, fondato su numerose indagini empiriche, appare fondato su una posizione ideologica. Non entro nel dettaglio dei testi dell'Ottocento, le cui idee sono sopravvissute fino agli anni 80 del secolo scorso e, come ho già detto, fino ad oggi, presso studiosi affascinati dai concetti nuovi. A Roma, dunque, la religione degli antenati, cioè quella delle comunità antiche, le città-stato, le famiglie e le associazioni 
di ogni genere, sarebbe morta. La nascita dell'Impero romano alla fine del I. s. a.C. avrebbe spinto gli individui a perseguire la loro ricerca d'infinito e di Dio. Un argomento esterno per spiegare la sparizione della religione di Stato sarebbe stato la morte del regime politico delle città-Stato. Però venivano anche citati culti come quello di Asclepios/Esculapio, il dio medico, per provare che gli individui si rifugiavano presso divinità che li aiutavano, apparentemente al di fuori del sistema dei culti pubblici. Tornerò su questo punto più avanti.

Un volume collettivo pubblicato nel 2013 col titolo The individualization in the Religions of the Ancient Mediterranean, riportava le ricerche fatte nell'Università di Erfurt. Nell'introduzione del volume il mio collega e amico Jörg Rüpke nega ogni legame fra la religione e una struttura collettiva tale la città, la città-Stato, poichè l'Impero avrebbe offerto un quadro generale a questa de-tradizionalizzazione religiosa tramite la libera circolazione degli abitanti del Mediterraneo romano, resa possibile dal I. al IV. secolo d.C. Secondo lui è l'individuo in quanto agente religioso che si deve studiare per spiegare questo cambiamento di cui gli storici dell'antichità e soprattutto gli storici delle religioni greca e romana non avrebbero mai tenuto conto. Incapaci di elevarsi al livello globale dei «religious studies», essi avrebbero negletto i culti privati, la magia e tutti i comportamenti che non entrerebbero nel quadro dei culti ufficiali. Non torno su questi argomenti già criticati nel mio libro del 2013. Tuttavia, ad eccezione di qualche ripetizione del dogma, non si trovano nel libro contributi che possano appoggiare questa posizione poco empirica; ma piuttosto degli studi di alcuni esponenti maggiori della storia delle religioni che sembrano contraddire questa teoria.

Ho parlato di Asclepios/Esculapio. Corinne Bonnet, per esempio, si interessa a questa divinità dopo la conquista macedonica dell'Asia Minore; più precisamente della Fenicia. Costatando che è impossibile distinguere nelle fonti fra atti religiosi collettivi e atti religiosi privati, $\mathrm{C}$. Bonnet descrive il culto del dio Eshmoun/Asclepios non come un culto personale, ma come una strategia cultuale dei suoi adoratori e un compromesso fra la tradizione fenicia e il nuovo mondo ellenistico. Eshmoun era tradizionalmente legato ai bambini, al loro sviluppo e alla loro integrazione nella collettività. In epoca ellenistica, Asclepios/Eshmoun si occupa delle stesse funzioni, e non c'è nessuna traccia nel suo culto di riti più individuali destinati al corpo, alla cura di sé stesso o addirittura al destino personale. Più che di un segno di individualizzazione e di ricerca di una religione personale, questo culto 
offre una testimonianza dello sviluppo di una nuova koinè religiosa. Un altro articolo, di Gregory Woolf, che ancora poco tempo prima si era espresso criticamente sulla cosiddetta polis religion, sottolinea che i riti individuali trasformano il rapporto personale con la società. Woolf conclude giustamente che la scelta individuale di ruoli religiosi a partire da modelli preesistenti, che può essere distinta della partecipazione passiva ai riti degli antenati, non corrisponde a quel che chiamiamo oggi religiosità personalizzata, e ancora meno corrisponde a quel tipo di impegno in una riflessione cosmologica ed etica, che è stata valorizzato o criticato dopo la Riforma. Ancora più importante mi pare lo studio di Fritz Graf, che nessuno può accusare di aver negletto le pratiche religiose individuali, poiché ha rilanciato, ormai trent'anni fa, lo studio della magia antica, la pratica rituale più personale che ci sia, in un libro che pubblicava i seminari che aveva tenuti all'Ecole pratique des hautes études presso la mia cattedra. In questo articolo Graf si propone come obiettivo di studiare l'opposizione culti pubblici/culti privati nei documenti epigrafici. Ha scelto un certo numero di fondazioni o di iniziative private volte a creare o restaurare dei luoghi di culto, in Italia e nel mondo greco. Sottolineando che in questo tipo di iniziativa il pensiero religioso è indipendente dalla pratica religiosa, un particolare che viene sempre dimenticato, egli dimostra che la pratica religiosa pone dei limiti fortissimi all'individualizzazione. Infatti, gli individui del mondo greco-romano agiscono, certo, in funzione di un'iniziativa privata, ma sempre in rapporto col gruppo e per integrarsi in questo. Nelle epigrafi - strumento importantissimo di comunicazione fra individui e gruppo questo legame con la collettività e dunque con la tradizione degli antenati risulta ancora più forte. Lo fanno capire le discussioni filosofiche dei membri dell'élite, all'ombra di una biblioteca, su questioni relative agli dei e al culto degli antenati.

Non voglio continuare le critiche al modello teorico proposto da Jörg Rüpke nel volume menzionato. Più importante mi pare la ricerca delle origini di tale teoria.

G. Woolf scrive nel volume citato che «la variabilità degli aspetti che la nozione del 'me' è capace di rivestire è un fatto ben conosciuto degli antropologi che hanno lavorato sulla nozione di persona», dopo l'articolo seminale di Marcel Mauss del 1938. Arnaldo Momigliano concedeva di non capire bene questa nozione francese della persona, e dava la colpa di questa incomprensione alla battaglia di San Quintino, nel 1557, che aveva fatto dei suoi antenati che vivevano a Montmélian, nel 
ducato di Savoia, dei sudditi dell'imperatore e non del re di Francia. Questa battuta descrive bene un aspetto del dibattito sull'individualizzazione, che sfugge spesso ai colleghi tedeschi, per i quali l'antropologia è una specie di fenomenologia ispirata dalla teologia cristiana e non corrisponde per niente all'antropologia sociale e religiosa che si è andata costituendo progressivamente, dall'inizio del Novecento, in Francia, Inghilterra e Stati uniti. Nella teoria citata, la nozione di individuo e di persona è semplicemente assimilata alla nostra concezione della persona e dell'individuo. Invece queste due concezioni sono estremamente diverse. Un convegno molto noto che ebbe luogo a Royaumont nel $1985 \mathrm{fu}$ dedicato a tutti gli aspetti dell'individuo. Per quanto riguarda gli antichisti, gli Atti del convegno contenevano un articolo di Jean-Pierre Vernant su «L'individuo nella città», un articolo che non viene menzionato nelle bibliografie del volume di J. Rüpke. Gallicum non legitur, diceva Claude Nicolet. Ora, una delle osservazioni di Vernant stabiliva che sul piano religioso la società greca non era gerarchica, ma egualitaria. Non c'era una casta sacerdotale, ma i ruoli sacerdotali erano ricoperti dai magistrati e dai cittadini stessi. Quest'integrazione nella comunità si produce in un quadro sociale in cui l'individuo non appare come un rinunciante, un asceta - Vernant allude qui all'articolo di Louis Dumont sull'India nello stesso convegno - ma come un soggetto di diritto, un attore politico, una persona privata nella sua famiglia o fra i suoi amici. Non entro nei dettagli. Vernant ricorda che il mondo greco conosce l'individuo stricto sensu quale emerge da una biografia, e d'altra parte la persona che si esprime tramite pratiche e attitudini psicologiche che conferiscono al soggetto una dimensione d'interiorità e d'unicità, la sua singolarità. Nella società antica esistono due campi, il collettivo, nel quale tutte le pratiche debbono essere condivise, e il campo privato in cui le pratiche non debbono essere condivise e riguardano soltanto ciascuno singolarmente. La collettività è rigida a Sparta, mentre ad Atene il privato, che è legato alla vita domestica, viene definito dai genitori e dai familiari in un'area dove i rapporti privati fra individui possono svilupparsi. Al contrario l'intimità del 'me' è ignorata dagli Antichi. Il 'me' non è né delimitato né unificato, non è il prodotto dell'introspezione, ma il riflesso delle sue attività ed opere. L'esistenza è prioritaria rispetto alla coscienza di esistere. Se l'individuo è valorizzato in quanto tale, nella sua singolarità, come Achille o un mago ispirato come Empedocle, questi è separato dai suoi eguali e dai suoi capi, ma questo particolare non ne fa un asceta. E l'anima di Socrate presso Platone non è per niente l'anima cristiana del 
futuro. Non è il 'me' di Socrate, è l'anima impersonale, supra-personale, l'anima in Socrate, in me, piuttosto che la sua anima, la mia anima. È un $\delta \alpha i ́ \mu \omega v$. Rimandando a Peter Brown, Vernant conclude che la cura di sé dei filosofi e le pratiche sociali corrispondenti daranno nascita a una nuova nozione di persona.

In questo nuovo stile di relazione col divino e di esperienza di sé, il modello di parità sparisce, ormai gli individui delegano a individui eccezionali la funzione di assicurare il legame fra cielo e terra. Agostino viene generalmente considerato come il primo individuo di questo tipo che sia ben conosciuto, e che segna la nascita della persona e dell'individuo nel senso in cui noi l'intendiamo dopo secoli di sviluppo. Per quanto ci riguarda, la situazione non cambia dall'epoca anteriore fino al IV-V secolo d.C., fino a Graziano e Teodosio, cioè fino al momento in cui le fonti segnalano problemi gravi nel funzionamento delle istituzioni della religione di Stato, anche se la religione ancestrale sopravvive per quasi un secolo in certe regioni del mondo romano e nelle famiglie. Questo mutamento però è storia, non una speculazione della filosofia romantica.

Detto questo, torniamo un attimo alla posizione difesa da J. Rüpke. Quello che dimentica completamente è la cittadinanza, quelle delle città dell'Impero e quella di Roma. Nella teoria dell'individualizzazione, l'individuo viene sostituito al cittadino, una confusione che esiste anche nel mondo liberale di segno inglese. Per i Francesi, anche se per i liberali Benjamin Constant o Numa Fustel de Coulanges la città antica era da considerare un regime totalitario, la cittadinanza costituisce un tratto essenziale dell'individuo nelle vita collettiva, gli attribuisce dei diritti e dei doveri che niente può compensare. L'individuo antico era prima di tutto un cittadino, con dei privilegi e dei doveri precisi. Un errore della stessa gravità è l'idea che sta dietro questa definizione della persona, cioè la presunzione che l'individuo privato facesse quello che voleva. Non ho bisogno di commentare a lungo questa visione. Tutti sanno che i membri della domus e della familia, così i liberi come gli schiavi, erano sotto il potere domestico del pater familias, e che non ci si sottraeva facilmente ai propri obblighi.

L'immagine generale della religione e dello spazio individuale che si sarebbe sviluppato a partire dalla fine del I sec. a. C. non è un'invenzione recente. Ho già accennato alla teoria della decadenza religiosa romana e possiamo riprenderla, per chiudere questo tema. Il manuale di religione romana pubblicato da Georg Wissowa nel 1902 suscitò una piccola polemica di cui l'introduzione della seconda edizione rende 
conto nel 1912. Ulrich von Wilamowitz-Moellendorf aveva giudicato severamente questo manuale, dicendo che mancava di ogni senso della religiosità e adottava soltanto la religio, il punto di vista dei sacerdoti romani, lasciando da parte la religione. Nella sua risposta Wissowa dice di dubitare che «Religiosität» - fra virgolette nel testo - fosse una realtà universale e rivendica chiaramente un altro approccio ai fatti religiosi. In effetti, la parola e la definizione di Religiosität usate da Wilamowitz provengono direttamente dal teologo Friedrich Schleiermacher, che dopo Lutero contrapponeva questo legame personale e diretto col Creatore alla chiesa, l'istituzione mediatrice fra individuo e Dio. Non entro nei dettagli. Queste posizioni sono ben conosciute. L'importante è che la filosofia e la storia del romanticismo tedesco hanno ripreso questa definizione della religione, che negava i riti e il magistero della Chiesa. Quasi tutti gli storici, compreso Theodor Mommsen, notoriamente agnostico, utilizzarono questo schema per descrivere la religione greca o romana. E questa teoria ha anche dato alla luce uno dei miti storiografici del Novecento: i culti orientali, che sarebbero la prima manifestazione di questa ricerca di una nuova Religiosität. Poco a poco queste idee si diffusero anche negli ambienti cattolici, ma nell'Ottocento non era così. Rimando alla reazione di Wissowa che era un cattolico della Slesia. Un esempio dell'influsso delle opinioni religiose è offerto dalle interpretazioni dei rendiconti dei Fratelli Arvali, un monumento del ritualismo romano. Gaetano Marini pubblicò nel 1795 un terzo dei testi conosciuti oggi; un secolo dopo, Wilhelm Henzen, che aveva fatto scavare il luogo di culto degli Arvali li aumentò di ben due terzi. Ma malgrado il numero limitato di testi conosciuti da Marini, le sue interpretazioni sono molto migliori di quelle di Henzen. Grande epigrafista, Henzen non capiva o piuttosto non si interessava affatto ai riti descritti dagli atti degli Arvali. Nel migliore dei casi citava Marini, nel peggiore rimaneva silenzioso. La ragione è semplice. Gaetano Marini era cattolico, e abituato a praticare, a vedere e a analizzare dei riti; Wilhelm Henzen era luterano e non si interessava ai riti degli Arvali. Non ci si sorprenderà allora del fatto che, quando una nuova tavola con una bella descrizione dei riti sacrificali degli arvali fu pubblicata nel 1914, Wissowa scoprì i riti arvalici e ne fece un commento eccellente.

Torno al nostro argomento. Avrete capito che l'insistenza di certi studiosi sull'individualizzazione religiosa e la morte del ritualismo romano riproduce, coscientemente o no, la dottrina teologica che risale al pensiero della Riforma. Così impongono alle fonti un'interpretazione 
forzata ed erronea. Le religioni romane erano certamente spregevoli per i padri della chiesa, che vi vedevano, come Lattanzio dice in una formula magnifica, ritum ad solos digitos pertinentem, «un modo rituale che riguarda unicamente le dita». Ma non dicono per niente che l'altra religione era morta. La combattevano, la criticavano, ma accettavano la sua realtà. L'alterità di questa religione irrita certi studiosi attuali, soprattutto nei paesi che non hanno conosciuto l'antropologia sociale. Una volta che uno studioso accetta questi obblighi religiosi, scopre nella documentazione che la religione ritualista dei Romani, tutt'altro che morta, in realtà fioriva e prosperava durante i tre primi secoli dell'Impero. Questi costumi non erano meno diffusi e vivaci rispetto a molte delle religioni attuali, presso gran parte dell'umanità. Riprendendo le parole di Georg Wissowa, dovremmo forse chiedere un po' di tolleranza per le religioni rituali, quelle degli Antichi e quelle di gran parte dell'umanità attuale. 
